\title{
HERENCIA DE LA POLIEMBRIONÍA EN DOS POBLACIONES EXPERIMETALES DE MAÍZ
}

\section{POLYEMBRYONY INHERITANCE IN TWO EXPERIMENTAL MAIZE POPULATIONS}

\author{
Hermes Rebolloza Hernández ${ }^{1}$, José Espinoza Velázquez ${ }^{2 *}$, Daniel Sámano Garduño \\ y Víctor M. Zamora Villa ${ }^{3}$
}

\begin{abstract}
${ }^{1}$ Postgrado en Fitomejoramiento, Universidad Autónoma Agraria Antonio Narro (UAAAN). ${ }^{2}$ Instituto Mexicano del Maíz "Dr. Mario E. Castro Gil”, UAAAN. Tel. y Fax: 01(844)411-0231 y 411-0309. ${ }^{3}$ Departamento de Fitomejoramiento, UAAAN. Calzada Antonio Narro Núm. 1923. 25315, Buenavista, Saltillo, Coahuila, México.

*Autor para correspondencia (jespvel@uaaan.mx)
\end{abstract}

\section{RESUMEN}

Aquí se plantea que la poliembrionía (PE) que ocurre en dos poblaciones de maíz (Zea mays L.) es controlada por dos loci epistáticos, donde basta la presencia de un alelo dominante de cualquiera de ellos para expresar la condición normal de plántula (acción génica duplicada), por lo que la poliembrionía se expresa sólo con el genotipo doble homocigótico recesivo. El análisis genético incluyó progenies $F_{1}, F_{2}$ y $R C_{1}$ de cruzas de las poblaciones IMMUAAAN-BAP (D) e IMM-UAAAN-NAP (C) con ocho materiales exóticos, los cuales fueron seis híbridos comerciales, un híbrido simple experimental y una población del CIMMYT. Las 16 cruzas $F_{1}$ fueron obtenidas en Tepalcingo, Mor., en 2007; las $F_{2}$ en Buenavista, Coah., en 2008; y las retrocruzas $\left(\mathbf{R C}_{1}\right)$ en Buenavista, en 2009. La evaluación de la $P E$ en progenies $F_{1}$ y $F_{2}$ se hizo en invernadero y campo, bajo un diseño de bloques completos al azar con 4 o 5 repeticiones, y la $R_{1}$ en invernadero. La segregación de la $P E$ en $F_{2}$ se probó con la hipótesis de dos loci recesivos hipostáticos 15:1 (progenie No-PE vs. progenie PE), y para las retrocruzas la hipótesis fue 12:4. Como se esperaba, la $\mathrm{PE}$ no se expresó en $F_{1}$ pero sí en $F_{2}$ y $R_{1}$, en proporciones de 3 a $7 \%$ y de 13 a $22 \%$, respectivamente. Estas proporciones son compatibles con las hipótesis planteadas, pero con la consideración de penetrancia incompleta la cual se pudo observar en montos de 10 a $50 \%$, según la fuente de germoplasma exótico en combinación con las poblaciones PE. Los resultados de pruebas de $\chi^{2}$ aplicadas a las proporciones observadas en los dos tipos de análisis estadístico, apoyan la propuesta de herencia de la $\mathrm{PE}$ en las dos poblaciones de maíz bajo estudio.

Palabras clave: Zea mays, herencia, análisis genético mendeliano.

\section{SUMMARY}

In this work it is postulated that polyembryony (PE) occurring in two maize populations (Zea mays L.) is under the control of two epistatic loci, where a dominant allele at either gene pair produces the normal seedling trait (duplicate gene action), so that polyembryony is shown only by the double homozygous recessive genotype. The genetic analysis was based on the $F_{1}, F_{2}$ and $B_{1}$ progenies from crosses among the populations IMM-UAAAN-BAP (D) and IMM-UAAAN-NAP (C) with eight non related germplasm sources, which were six commercial hybrids, one experimental simple hybrid and a population from CIMMYT. The $16 F_{1}$ crosses were done in 2007 in Tepalcingo, Mor; the $F_{2}$ during 2008, in Buenavista, Coah., and The back crosses $\left(\mathrm{BC}_{1}\right)$ were generated in 2009, in Buenavista. The PE evaluation for $F_{1}$ and $F_{2}$ was done under both greenhouse and field conditions using a complete block design, with 4 or 5 replicates; the $\mathrm{BC}_{1}$ evaluations were performed only under greenhouse. The PE segregation in $F_{2}$ was tested under the hypothesis of two recessive hypostatic loci, ratio 15:1 (No-PE vs $\mathrm{PE}$ progenies); for the $\mathrm{BC}_{1}$ the hypothesis was for the 12:4 ratio. As expected, the PE was not shown in $\mathrm{F}_{1}$ progenies, but it was observed in $F_{2}$ and $B_{1}$, with proportions of 3 to $7 \%$ and 13 to $22 \%$, respectively. These results are compatible with the proposed hypothesis, considering a joint effect with incomplete penetrance; this phenomenon was present in amounts of 10 to $50 \%$, varying according to the exotic germplasm in use. The applied $\chi^{2}$ tests to the two types of hypothesis support the proposed inheritance pattern for $P E$ in the two studied maize populations.

Index words: Zea mays, inheritance, Mendelian genetic analysis.

\section{INTRODUCCIÓN}

La poliembrionía (PE) en maíz (Zea mays L.) ha sido reportada como un fenómeno de herencia simple y recesiva (Morgan y Rappleye, 1951; Kermicle, 1971; Pilu, 2000; Evans, 2007), pero también como de herencia cuantitativa (Pesev et al., 1976; Espinoza et al., 1998).

El caso más estudiado de $\mathrm{PE}$ es el gen mutante igl (indeterminate gamethophyte), de efectos pleiotrópicos, que puede generar poliembrionía (6\%), progenie monoploide $(3 \%)$ y casos de embriones en miniatura 
(>1 \%) (Kermicle, 1969; 1971; Hallauer y Miranda, 1988; Evans, 2007).

Ciertas clases de PE en maíz pueden originarse de varias maneras, como mediante la formación de gemelos o tripletes a partir de célula huevo pro-embrionaria (cleavage), o de sacos embrionarios múltiples en un óvulo, e incluso por inducción con la fitohormona 2-4-D en sacos embrionarios 2 d después de la polinización (Erdelska y Vidovencova, 1992; Erdelska, 1996).

Aplicaciones agronómicas de la PE en maíz, sin incluir la que pudiera causar el mutante igl, ha sido informada en la formación de líneas endogámicas (Pesev et al., 1976) y en la aplicación de selección recurrente para incrementar su frecuencia (Espinoza et al., 1998). Estos últimos autores reportan el origen de una población de maíz con el carácter "plantas gemelas", a la que en sus primeros ciclos de selección se le determinó una heredabilidad de $65 \%$ por la vía progenie-progenitor promedio.

La PE que aquí se informa es la que puede ser observada en dos poblaciones de maíz, una de porte alto (NAP o C) y otra enana (BAP o D), ambas derivadas de la población base que mencionan Espinoza et al. (1998) y en las que actualmente el carácter se concentra en frecuencias promedio superiores a $50 \%$. En estas poblaciones, la PE se considera como un carácter que potencia el rendimiento y la calidad nutrimental del grano, porque genera dos o más plantas completas por semilla, contiene de 30 a $50 \%$ más grasa cruda, 15 a 20 $\%$ más ácido graso oleico, y 20 a $50 \%$ más de lisina que el maíz común (Espinoza et al., 1998; Valdés, 2005, com. personal ${ }^{1}$; González, 2009, com. personal²).

El potencial de utilización de esta PE ha motivado el replanteamiento de los mecanismos de herencia que la controlan, pues en un principio fue considerada como de herencia cuantitativa (Espinoza et al., 1998) y con respuesta a la selección (Espinoza $\mathrm{V} \mathrm{J}$, datos propios no publicados), y ha sido imposible constituir una población totalmente poliembriónica aun después de 20 ciclos de selección recurrente. En las poblaciones bajo observación, algunas familias pueden exhibir frecuencias

\footnotetext{
${ }^{1}$ Valdez L E L (2005) Ganancia en calidad nutrimental del grano como respuesta asociada a la selección para poliembrionía en maíz. Tesis de maestría en ciencias en Fitomejoramiento, Universidad Autónoma Agraria Antonio Narro (UAAAN). Buenavista, Saltillo, Coahuila, México. 92 p.

${ }^{2}$ González V V M (2009) Formación y caracterización de grupos germoplásmicos de maíz, poliembriónicos y alto contenido de aceite. Tesis de doctor en ciencias en Fitomejoramiento, UAAAN. Buenavista, Saltillo, Coahuila, México. 69 p.
}

de hasta $80 \%$, pero la frecuencia promedio de población general es de 50 a $65 \%$; incluso, la aplicación de autofecundaciones sucesivas $\left(\mathrm{S}_{3}\right)$ en varias líneas de las dos poblaciones exhiben una conducta variable en el monto de las frecuencias PE, con valores que oscilan de 45 a $85 \%$ (datos propios no publicados).

Con la experiencia acumulada en el manejo de estas dos poblaciones $(\mathrm{C}$ y $\mathrm{D})$, aquí se presenta un replanteamiento de la naturaleza genética de la $\mathrm{PE}$, y se propone un patrón de herencia mendeliano por la acción de dos loci, con interacción epistática del tipo 15:1 en segregación $\mathrm{F}_{2}$, con una penetrancia incompleta del orden de 10 y hasta $50 \%$, esto en función de la combinación del germoplasma PE con otras fuentes ajenas a C y D.

\section{MATERIALES Y MÉTODOS}

Se utilizaron dos poblaciones poliembriónicas (PE), IMM-UAAAN-BAP (D) e IMM-UAAAN-NAP (C), más ocho genotipos No-PE: la cruza simple CS8xML (Mat-1), la población Tuxpeño-HO (Mat-2), ésta facilitada por el Centro Internacional de Mejoramiento de Maíz y Trigo (CIMMYT) y seis híbridos comerciales [de Pionner: 30G54 (Com-1); de Asgrow: Leopardo (Com-2), Oso (Com-3) y Puma (Com-6); de Dekalb: D2020 (Com-4) y D2002 (Com-5)]. Las cruzas entre poblaciones PE (polinizadores) con los genotipos No-PE generaron 16 grupos germoplásmicos $\mathrm{F}_{1}$, y a partir de ellos se produjeron los correspondientes grupos $\mathrm{F}_{2}$ y la retrocruzas $\left(\mathrm{RC}_{1}\right)$ de las $\mathrm{F}_{1}$ con los progenitores $\mathrm{D}$ y $\mathrm{C}$.

El establecimiento y manejo de los grupos $\mathrm{F}_{1}, \mathrm{~F}_{2}$ y $\mathrm{RC}_{1}$ se llevó a cabo en condiciones de campo e invernadero en Buenavista, Coah. [ $\left[25^{\circ} 21^{\prime} \mathrm{LN}, 101^{\circ} 02^{\prime} \mathrm{LO}, 1756 \mathrm{~m}\right.$ de altitud (CETENAL, 1975)] y en el campo experimental "Dr. Mario E. Castro Gil", en Tepalcingo, Mor. (18 26’ LN, 98 $18^{\circ}$ ' LO y altitud de $1100 \mathrm{~m}$ ).

Las progenies $F_{1}$ se generaron en el ciclo OtoñoInvierno (O-I) 2007/08 en Tepalcingo. A partir de éstas se siguieron dos estrategias de cruzamiento; una de ellas fue generar las $F_{2}$ por cruzamientos fraternales planta a planta dentro de cada grupo; la otra, cruzar a las $\mathrm{F}_{1}$ con las poblaciones D y C para generar las retrocruzas $\left(\mathrm{RC}_{1}\right)$. Estos procesos se llevaron a cabo en Buenavista, en los ciclos Primavera-Verano (P-V) 2008 y P-V 2009, respectivamente.

Las evaluaciones de plántulas en invernadero de todos los grupos $\left(\mathrm{F}_{1}, \mathrm{~F}_{2}\right.$ y $\left.\mathrm{RC}_{1}\right)$, se llevaron a cabo en Buenavista durante el verano y otoño 2009, y como testigos se agregaron muestras de las poblaciones D y C. Las evaluaciones en condiciones de campo se llevaron a 
cabo de manera progresiva, conforme se fueron generando los grupos, en Buenavista (P-V 2008), en Tepalcingo (O-I 2008/2009), y en Buenavista (P-V 2009).

El número de semillas sembradas en invernadero fue: progenies $F_{1}$, dos repeticiones de 100 ; y progenies $F_{2}$ y $\mathrm{RC}_{1}$, con cinco repeticiones de 200 . En campo la muestra fue: progenies $F_{1}, 462$ semillas (154 por repetición) distribuidas en siete surcos de $5 \mathrm{~m}$; para las $\mathrm{F}_{2}$ evaluadas en Tepalcingo, 5000 semillas (12 surcos de $18 \mathrm{~m}$ ); en Buenavista, 6000 semillas (12 surcos de $22 \mathrm{~m}$ ). En toda siembra de campo la distancia entre surcos fue $0.8 \mathrm{~m}$. El manejo de los lotes fue con riego, fertilización de $80 \mathrm{~N}$ $80 \mathrm{P}-0 \mathrm{~K}$, con control químico de plagas y con combate químico-mecánico de malezas.

El diseño experimental utilizado en todos los casos fue uno de bloques completos al azar. El número de repeticiones en las evaluaciones de invernadero fue: dos en las progenies $F_{1}$, y cinco para $F_{2}$ y $R_{1}$. En campo, las repeticiones fueron tres para progenies $\mathrm{F}_{1} \mathrm{y}$ cuatro para progenies $\mathrm{F}_{2}$.

Las variables de respuesta fueron: porcentaje de emergencia (FE), porcentaje de poliembrionía (FPE) y porcentaje de plántulas anormales (FPA). Las mediciones en plántulas $\mathrm{F}_{1}, \mathrm{~F}_{2}$ y $\mathrm{RC}_{1}$ se llevaron a cabo a las dos semanas después de la siembra en invernadero, y a las tres semanas en condiciones de campo. Los datos de plántulas poliembriónicas en relación con el total de plántulas emergidas en cada grupo $\left(\mathrm{F}_{1}, \mathrm{~F}_{2} \mathrm{o} \mathrm{RC}_{1}\right)$, de campo o invernadero, se utilizaron para probar las hipótesis que se propusieron para filiales y retrocruzas, mediante pruebas de $\chi^{2}$.

La hipótesis propuesta para la segregación $\mathrm{F}_{2}$ corresponde a las clases fenotípicas "progenie normal, No-PE" vs. "progenie PE", en proporción de 15:1, como se espera en el caso de dos loci, con dos alelos cada uno y dominancia completa en cada par, donde basta la presencia de un alelo dominante de cualquiera de ellos para expresar el carácter normal, es decir, No-PE; dicho de otra forma, la condición PE se genera únicamente con la acción de los alelos recesivos de los dos loci en homocigosis. Por otra parte, la hipótesis para las progenies $\mathrm{RC}_{1}$, bajo la suposición de las mismas causas genéticas, es de 12:4 para las dos clases fenotípicas esperadas. Dado que la prueba de $\chi^{2}$ fue realizada para cada repetición y grupo genotípico, se procedió a efectuar una prueba de homogeneidad para validar el uso de datos globales a través de repeticiones.

El análisis de varianza para FE, FPE y FPA, cuyos datos tuvieron transformación angular, se hizo con el paquete estadístico SAS (SAS Institute, 1999). La varianza de la fuente "cruzas" se partió en sus componentes: población $\mathrm{C}$ y población $\mathrm{D}$, e incluyen el contraste D vs. C, con la finalidad de conocer el desempeño de $\mathrm{C}$ y $\mathrm{D}$ como polinizadores. La prueba de contrastes fue mediante una prueba de " $\mathrm{t}$ " $(\mathrm{P}<0.01)$.

\section{RESULTADOS Y DISCUSIÓN}

Los promedios de variables en los grupos $\mathrm{F}_{1}$ que se muestran en el Cuadro 1 indican que la germinación fue 4 a $15 \%$ más alta en invernadero que en campo, y que aun en campo la germinación fue igual o superior a $81 \%$. Este resultado agronómicamente deseable debe abonarse a la hibridación entre PE y materiales exóticos, ya que la germinación reportada de PE en campo había sido históricamente baja (Espinoza et al. 1998).

Como se esperaba, la $\mathrm{F}_{1}$ no exhibió $\mathrm{PE}$, con excepción de los datos observados en C y D utilizadas como testigos, cuyas frecuencias fueron superiores a $55 \%$ en invernadero y a $50 \%$ en campo; estos valores representan el promedio actual del carácter en estas poblaciones. La ausencia de PE en las $\mathrm{F}_{1}$ evidencia que el carácter no es cuantitativo como lo reportaron Espinoza et al. (1998), pero sí de naturaleza recesiva como se propone en la presente investigación, y que del maíz normal germina una sola plántula por semilla.

Las variables medidas en los grupos $\mathrm{F}_{2}$ crecidas en invernadero (Cuadro 2) nuevamente muestran germinación alta $(>85 \%)$ y similar en todos los cruzamientos con $\mathrm{C}$ o D, con excepción de la cruza Com$5 \times$ D que superó $(\mathrm{P}<0.01)$ a la cruza Com-5 x C. En todas las progenies $\mathrm{F}_{2}$ reaparece el carácter $\mathrm{PE}$, con una gama de frecuencias que va de 3.0 a $7.1 \%$, variación asociada con el germoplasma exótico involucrado. Del conjunto de ocho materiales en hibridación con $\mathrm{PE}$, únicamente la fuente Com-1 consistentemente redujo la expresión del carácter, sea en cruza con $\mathrm{C}$ o $\mathrm{D}$, con una frecuencia promedio de $3.2 \%$. De las otras combinaciones sobresalen los segregantes de cruzamientos PE x Com-2 y Com-4, cuyas frecuencias observadas fueron 5.99 y $6.15 \%$, valores prácticamente iguales a las proporciones esperadas de 1/16. También, todas las combinaciones de $\mathrm{C}$ o $\mathrm{D}$ por cualquier otro material ajeno a ellas, resultaron estadísticamente iguales, con frecuencias PE promedio de $5 \%$ aproximadamente, con excepción del caso de Com-5 (P < 0.01). La PE en las poblaciones como tales, evaluadas en simultáneo con los grupos segregantes $\mathrm{F}_{2}$, presentaron frecuencias de un poco más de $50 \%$, proporción acorde con sus valores mínimos esperados. 
Cuadro 1. Promedios de germinación y poliembrionía (PE) de maíz en progenies $F_{1}$ de las poblaciones $D$ y $C$, en invernadero y campo. Evaluación en Buenavista, Coah. 2008.

\begin{tabular}{|c|c|c|c|c|c|c|c|c|}
\hline \multirow{3}{*}{ Grupos } & \multicolumn{4}{|c|}{${ }^{\dagger} \mathrm{F}_{1}$ Invernadero } & \multicolumn{4}{|c|}{${ }^{\dagger \dagger} \mathrm{F}_{1}$ Campo } \\
\hline & \multicolumn{2}{|c|}{ Plántulas emergidas } & \multicolumn{2}{|c|}{ Plántulas PE } & \multicolumn{2}{|c|}{ Plántulas emergidas } & \multicolumn{2}{|c|}{ Plántulas PE } \\
\hline & $\mathrm{D}$ & $\mathrm{C}$ & $\mathrm{D}$ & $\mathrm{C}$ & $\mathrm{D}$ & $\mathrm{C}$ & $\mathrm{D}$ & $\mathrm{C}$ \\
\hline Com-1 & $97 \pm 1$ & $99 \pm 1$ & 0 & 0 & $136 \pm 8$ & $128 \pm 16$ & 0 & 0 \\
\hline Com-2 & $96 \pm 4$ & $98 \pm 1$ & 0 & 0 & $134 \pm 14$ & $128 \pm 18$ & 0 & 0 \\
\hline Com-3 & $97 \pm 2$ & $97 \pm 1$ & 0 & 0 & nd & nd & nd & nd \\
\hline Com-4 & $99 \pm 1$ & $95 \pm 3$ & 0 & 0 & nd & nd & nd & nd \\
\hline Com-5 & $93 \pm 3$ & $86 \pm 3$ & 0 & 0 & $126 \pm 19$ & $126 \pm 17$ & 0 & 0 \\
\hline Com-6 & $99 \pm 1$ & $100 \pm 0$ & 0 & 0 & $133 \pm 15$ & $145 \pm 20$ & 0 & 0 \\
\hline Mat-1 & $97 \pm 1$ & $88 \pm 1$ & 0 & 0 & nd & nd & nd & nd \\
\hline Mat-2 & $96 \pm 3$ & $96 \pm 1$ & 0 & 0 & nd & nd & nd & nd \\
\hline Media general & $97 \pm 4$ & $95 \pm 10$ & 0 & 0 & $130 \pm 19$ & $131 \pm 18$ & 0 & 0 \\
\hline Polinizadores & $92 \pm 2$ & $97 \pm 6$ & $59 \pm 8$ & $74 \pm 6$ & $135 \pm 13$ & $127 \pm 12$ & $85 \pm 5$ & $80 \pm 6$ \\
\hline
\end{tabular}

†Tamaño de muestra $=200$ semillas, 100 semillas por repetición; ${ }^{\dagger \dagger}$ Tamaño de muestra $=462$ semillas, 154 semillas por repetición; nd $=$ no disponible.

Cuadro 2. Promedios de germinación, poliembrionía (PE) y anormalidades en plántulas segregantes $F_{2}$ de maíz evaluados en invernadero, y significancia de " $t$ " al comparar el efecto de D vs. C como polinizadores dentro de cada grupo. Buenavista, Coah. 2009.

\begin{tabular}{|c|c|c|c|c|c|c|}
\hline \multirow{3}{*}{ Grupo } & \multicolumn{6}{|c|}{$\mathrm{F}_{2}$} \\
\hline & \multicolumn{2}{|c|}{ Plántulas emergidas } & \multicolumn{2}{|c|}{ Plántulas PE } & \multicolumn{2}{|c|}{ Plántulas anormales } \\
\hline & $\mathrm{D}$ & $\mathrm{C}$ & $\mathrm{D}$ & $\mathrm{C}$ & $\mathrm{D}$ & $\mathrm{C}$ \\
\hline Com-1 & $195 \pm 2$ & $184 \pm 14 *$ & $6 \pm 2$ & $6 \pm 2$ & $2 \pm 1$ & $3 \pm 1$ \\
\hline Com-2 & $193 \pm 3$ & $191 \pm 6$ & $11 \pm 3$ & $12 \pm 2$ & $3 \pm 1$ & $3 \pm 3$ \\
\hline Com-3 & $197 \pm 3$ & $195 \pm 3$ & $6 \pm 2$ & $8 \pm 4$ & $2 \pm 1$ & $3 \pm 2$ \\
\hline Com-4 & $195 \pm 5$ & $195 \pm 2$ & $11 \pm 1$ & $13 \pm 3$ & $3 \pm 1$ & $6 \pm 1 * *$ \\
\hline Com-5 & $197 \pm 2$ & $170 \pm 9 * *$ & $7 \pm 3$ & $12 \pm 1 * *$ & $5 \pm 1$ & $9 \pm 1 * *$ \\
\hline Com-6 & $191 \pm 7$ & $185 \pm 16$ & $8 \pm 1$ & $7 \pm 3$ & $1 \pm 1$ & $6 \pm 5^{* *}$ \\
\hline Mat-1 & $193 \pm 5$ & $186 \pm 7$ & $9 \pm 2$ & $11 \pm 2$ & $5 \pm 1$ & $2 \pm 2$ \\
\hline Mat-2 & $193 \pm 3$ & $195 \pm 3$ & $9 \pm 3$ & $8 \pm 1$ & $4 \pm 1$ & $3 \pm 1$ \\
\hline Media general & $194 \pm 4$ & $188 \pm 11$ & $9 \pm 3$ & $10 \pm 3$ & $3 \pm 2$ & $4 \pm 3$ \\
\hline Polinizador, D o C & $185 \pm 8$ & $174 \pm 16$ & $128 \pm 9$ & $125 \pm 33$ & $23 \pm 6$ & $10 \pm 1$ \\
\hline
\end{tabular}

Tamaño de muestra $=1000$ semillas, 200 por repetición; $*$ y $* *=$ Prueba de $\mathrm{t}(\mathrm{P} \leq 0.05$ y 0.01 , respectivamente).

Cuadro 3. Promedios de retrocruzas $\left(\mathrm{RC}_{1}\right)$ hacia poblaciones poliembriónicas (PE) de maíz evaluadas en invernadero, y significancia de "t comparar el efecto de D vs. C como polinizadores dentro de cada grupo. Buenavista, Coah. 2009.

\begin{tabular}{|c|c|c|c|c|c|c|}
\hline \multirow{3}{*}{ Grupo } & \multicolumn{6}{|c|}{ Retrocruzas } \\
\hline & \multicolumn{2}{|c|}{ Plántulas emergidas } & \multicolumn{2}{|c|}{ Plántulas PE } & \multicolumn{2}{|c|}{ Plántulas anormales } \\
\hline & $\mathrm{D}$ & $\mathrm{C}$ & $\mathrm{D}$ & $\mathrm{C}$ & $\mathrm{D}$ & $\mathrm{C}$ \\
\hline Com-1 & $182 \pm 3$ & $187 \pm 4$ & $27 \pm 2$ & $37 \pm 4 * *$ & $15 \pm 2$ & $18 \pm 2$ \\
\hline Com-2 & $183 \pm 6$ & $182 \pm 2$ & $31 \pm 4$ & $34 \pm 3$ & $19 \pm 3$ & $16 \pm 4$ \\
\hline Com-3 & $188 \pm 4$ & $172 \pm 5^{* *}$ & $25 \pm 2$ & $35 \pm 8^{* *}$ & $17 \pm 5$ & $19 \pm 4$ \\
\hline Com-4 & $194 \pm 1$ & $193 \pm 3$ & $35 \pm 5$ & $42 \pm 4 * *$ & $15 \pm 4$ & $18 \pm 4$ \\
\hline Com-5 & $188 \pm 3$ & $184 \pm 4$ & $27 \pm 5$ & $28 \pm 2$ & $18 \pm 6$ & $16 \pm 4$ \\
\hline Com-6 & $190 \pm 3$ & $177 \pm 7^{* *}$ & $37 \pm 5$ & $28 \pm 3^{*}$ & $15 \pm 4$ & $22 \pm 2 * *$ \\
\hline Exp-1 & $185 \pm 6$ & $176 \pm 5^{* *}$ & $30 \pm 10$ & $34 \pm 5^{*}$ & $17 \pm 5$ & $27 \pm 4 * *$ \\
\hline Exp-2 & $191 \pm 2$ & $191 \pm 3$ & $30 \pm 5$ & $41 \pm 5^{* *}$ & $17 \pm 4$ & $17 \pm 4$ \\
\hline Media general & $188 \pm 5$ & $183 \pm 8$ & $30 \pm 6$ & $35 \pm 6$ & $17 \pm 4$ & $19 \pm 5$ \\
\hline Polinizador, D o C & $178 \pm 16$ & $178 \pm 4$ & $96 \pm 4$ & $90 \pm 12$ & $27 \pm 5$ & $32 \pm 4$ \\
\hline
\end{tabular}

Tamaño de muestra $=1000$ semillas, 200 por repetición; $*$, ** $=$ Prueba de $\mathrm{t}(\mathrm{P} \leq 0.05$ y 0.01 , respectivamente).

El promedio de "plántulas anormales" fue bajo (3 a 4 $\%)$, aunque estadísticamente superior $(\mathrm{P}<0.01)$ en la cruza de $\mathrm{C}$ con tres de los grupos comerciales (Com-5, -6 y -4), pero aún así fueron menores a $6 \%$; esto probablemente haya sido favorecido por la hibridación.

La germinación de las $\mathrm{RC}_{1}$ evaluadas en invernadero (Cuadro 3) fue superior a $85 \%$, a pesar de que en esta condición hay una proporción mayor de germoplasma poliembriónico (75:25, PE:No-PE). También resalta que tres de los ocho grupos presentaron mayor $(\mathrm{P}<0.01)$ germinación cuando se cruzaron con $\mathrm{D}$ que con $\mathrm{C}$, lo que perfila a D como la fuente de mejor aptitud para la hibridación. En las $\mathrm{RC}_{1}$ la recuperación de la PE se presentó en proporciones de 13 a $22 \%$, donde sobresalen por sus valores mayores $(\mathrm{P}<0.05$ y 0.01$)$ los 
retrocruzamientos hacia $\mathrm{C}$ en seis de los ocho materiales No-PE, con frecuencias PE de 16 a $22 \%$, que son las más cercanas a la proporción esperada de 4/16. Las frecuencias en los testigos $\mathrm{C}$ y $\mathrm{D}$ fueron ligeramente superiores a $50 \%$.

El número de plántulas anormales fue mayor que las observadas en $\mathrm{F}_{2}$, y más cercanas a las proporciones observadas en las poblaciones PE. La naturaleza del carácter produce anormalidades en plántula que obstruye su desarrollo; generalmente, las anormalidades en $\mathrm{C}$ y D se presentan en proporciones de 6 a $15 \%$, lo que pudiera explicar el monto promedio de $10 \%$ en el total de grupos $\mathrm{RC}_{1}$.

La evaluación de los grupos $\mathrm{F}_{2}$ en campo, en promedio de dos localidades (Cuadro 4) y de cuatro grupos segregantes, mostró que la germinación fue superior a 84 $\%$, no obstante que en estas condiciones se espera una baja germinación, particularmente cuando se incluyen progenitores PE (Espinoza et al., 1998). La presencia de la $\mathrm{PE}$ en los grupos $\mathrm{F}_{2}$ evaluados en campo varió de 3.1 a $5.4 \%$ sin diferencias por influencia del polinizador. Al igual que los resultados de invernadero en campo el progenitor No-PE Com-1 produjo la progenie con PE (3.55\%), algo menor que el progenitor Com-2 (4.65\%), diferencia que no fue significativa. Esta condición, reiterada en campo e invernadero, permite suponer que al combinar germoplasma PE con No-PE, la progenie puede expresarse con o sin obstrucción, en función del tipo de germoplasma acompañante.

El análisis de varianza para los grupos $\mathrm{F}_{2}$ evaluados en dos localidades en campo (Cuadro 5), que incluye la desagregación de la fuente "cruzas" en la primera localidad, permitió detectar que la PE de la población D (enana) es recibida de manera significativa y desigual por las diferentes fuentes de germoplasma exótico No-PE, lo que corrobora las tendencias detectadas en el comportamiento de los segregantes para Com-1 y Com-2. La frecuencia general de la PE en campo (último renglón de Cuadro 5) fue de $4 \%$, la cual representó sólo $64 \%$ de la proporción esperada de $6.25 \%$ según la hipótesis propuesta; con los datos obtenidos en esta muestra de combinaciones entre materiales PE y No-PE, la interferencia promedio en la expresión del carácter parece ser del orden de $36 \%$.

Según los análisis de varianza para la $\mathrm{PE}$ en estado de plántula de los grupos $\mathrm{F}_{2}$ y $\mathrm{RC}_{1}$ evaluados en invernadero
(Cuadro 6), también hay diferencias entre cruzas. La descomposición de esta fuente de variación en función de las poblaciones polinizadoras, permitió detectar que la frecuencia PE puede variar en función del germoplasma exótico combinado. Dada la proporción mayor de germoplasma $\mathrm{PE}$ en las $\mathrm{RC}_{1}$ también se detectaron diferencias $(\mathrm{P} \leq 0.05)$ entre los polinizadores $(\mathrm{D}$ vs. $\mathrm{C})$ al combinarse con el germoplasma exótico, diferencias que fueron más notorias cuando se cruzaron con la fuente $\mathrm{C}$.

Las pruebas de $\chi^{2}$ aplicada a los valores observados en los grupos $\mathrm{F}_{2}$ y $\mathrm{RC}_{1}$ (Cuadro 7) arrojaron datos relevantes en las pruebas de hipótesis de este trabajo; $i$. e., 15/16:1/16 (plantas normales, PN : plantas poliembriónicas, PE) en las $\mathrm{F}_{2}$, y 12/16:4/16 (PN:PE) en las $\mathrm{RC}_{1}$. Siete de los 28 casos $\mathrm{F}_{2}$ respondieron de acuerdo con lo esperado, ya que la segregación de la PE fue de $6.25 \%$, es decir que no presentaron obstrucción alguna en la expresión del carácter. Esto resultó igual con el polinizador $\mathrm{D}$ o $\mathrm{C}$ en $\mathrm{F}_{1}$, con excepción de Com-5 donde se acepta la hipótesis sólo para el polinizador C. Las pruebas de $\chi^{2}$ en los otros 21 casos $\mathrm{F}_{2}$ proporcionaron argumentos para rechazar las hipótesis, ya que las proporciones observadas del fenotipo PE fueron menores a lo esperado (Cuadro 2), las cuales se ubicaron en un rango de 3 a $5 \%$; el déficit resultante con respecto a 6.25 $\%$ pudiera deberse al fenómeno de penetrancia incompleta. En promedio, la reducción en la expresión de la $\mathrm{PE}$ en los casos estudiados fue de $33 \%$, valor que pudiera representar el monto de la penetrancia incompleta del carácter. Este monto podría ser mayor o menor, en función de la fuente del material exótico utilizado.

Las pruebas de $\chi^{2}$ en las $16 \mathrm{RC}_{1}$ (Cuadro 7) permiten señalar que las proporciones observadas fueron menores que las esperadas; el argumento central aquí es similar al observado en los 21 casos estadísticamente significativos de $F_{2}$, ya que el rechazo de la hipótesis fue por un déficit en la proporción segregante $\mathrm{PE}$, atribuible a la penetrancia incompleta del caracter. Las proporciones observadas se ubicaron en el rango de 13 a $21 \%$ (Cuadro 3 ), en lugar del $25 \%$ esperado. Por otra parte, la prueba de $\mathrm{t}(\mathrm{P}<0.05,<0.01)$ aplicada a la comparación entre polinizadores $\mathrm{C}$ y $\mathrm{D}$ (Cuadro 3) permitió detectar diferencias significativas en seis de los ocho casos analizados. La condición normal en altura de planta en $\mathrm{C}$ parece favorecer la expresión del carácter. 
Cuadro 4. Promedios de progenies $F_{2}$ de maíz poliembriónico (PE) en condiciones de campo y significancia de "t" para comparar el efecto del polinizador.

\begin{tabular}{|c|c|c|c|c|}
\hline \multirow[b]{2}{*}{ Grupo } & \multicolumn{2}{|c|}{${ }^{\dagger} \mathrm{F}_{2}$} & \multicolumn{2}{|c|}{${ }^{\dagger \dagger} \mathrm{F}_{2}$} \\
\hline & Plántulas emergidas & Plántulas PE & Plántulas emergidas & Plántulas PE \\
\hline \multirow{2}{*}{ Com-1 } & x D $1118 \pm 113$ & $35 \pm 6$ & $1233 \pm 20$ & $51 \pm 3$ \\
\hline & x C $1042 \pm 36$ & $42 \pm 4$ & nd & $\overline{\mathrm{nd}}$ \\
\hline \multirow{2}{*}{ Com-2 } & x D $1156 \pm 84$ & $62 \pm 8^{* *}$ & $1277 \pm 174$ & $68 \pm 9$ \\
\hline & x C $1201 \pm 26$ & $47 \pm 2$ & $\overline{\mathrm{nd}}$ & $\overline{\mathrm{nd}}$ \\
\hline \multirow{2}{*}{ Com-5 } & x D $1058 \pm 101^{*}$ & $48 \pm 4$ & $1366 \pm 135$ & $61 \pm 5$ \\
\hline & x C $1184 \pm 53$ & $55 \pm 4$ & nd & nd \\
\hline \multirow{2}{*}{ Com-6 } & x D $772 \pm 23^{*}$ & $28 \pm 5^{* *}$ & $1203 \pm 23$ & $51 \pm 7$ \\
\hline & x C $1051 \pm 86$ & $50 \pm 11$ & nd & nd \\
\hline \multirow{2}{*}{ Media general } & x D $1026 \pm 174$ & $43 \pm 14$ & $1270 \pm 118$ & $58 \pm 9$ \\
\hline & x C $1119 \pm 90$ & $49 \pm 7$ & nd & nd \\
\hline \multirow{2}{*}{ Polinizador } & $\mathrm{x} \mathrm{D} \quad \mathrm{nd}$ & nd & $1281 \pm 153$ & $825 \pm 61$ \\
\hline & x C & nd & $1231 \pm 125$ & $788 \pm 73$ \\
\hline
\end{tabular}

${ }^{\dagger}$ Evaluación O-I, 2008/09 en Tepalcingo, 1250 semillas por repetición (con excepción de Com-6 x D, sólo 800 semillas). ${ }^{\dagger}$ Evaluación P/V, 2009 en Saltillo, 1500 semillas por repetición; *,** $=$ Prueba de $\mathrm{t}(\mathrm{P} \leq 0.05$ y 0.01 , respectivamente). nd $=$ no disponible.

Cuadro 5. Factores de variación (FV), cuadrados medios (CM), grados de libertad (gl) y significancia para poliembrionía (PE) en maíz por ambiente y combinado, para los grupos $F_{2}$ evaluados en campo. Tepalcingo, Mor. 2008/2009 y Buenavista, Coah. 2009.

\begin{tabular}{|c|c|c|c|c|c|c|c|}
\hline \multirow[b]{2}{*}{ FV } & \multicolumn{2}{|c|}{ Tepalcingo, Mor. } & \multicolumn{3}{|c|}{ Buenavista, Coah. } & \multicolumn{2}{|c|}{ Combinado } \\
\hline & $\mathrm{gl}$ & $\mathrm{CM}$ & $\mathrm{gl}$ & $\mathrm{CM}$ & FV & $\mathrm{gl}$ & $\mathrm{CM}$ \\
\hline Repeticiones (R) & 3 & 0.00002 & 3 & 0.00001 & Localidades (L) & 1 & 0.00011 \\
\hline Cruzas & 7 & $0.00018^{* *}$ & 3 & $0.00012 *$ & $\mathrm{R} / \mathrm{L}$ & 6 & 0.00002 \\
\hline Población D & 3 & $0.00036^{* *}$ & & & Cruza (C) & 3 & $0.00044 * *$ \\
\hline Población C & 3 & 0.00005 & & & $\mathrm{C} \times \mathrm{L}$ & 3 & 0.00005 \\
\hline D vs. C & 1 & 0.00002 & & & & & \\
\hline Error & 21 & 0.00004 & 9 & 0.00002 & Error & 18 & 0.00003 \\
\hline CV (\%) & & 15 & & 11 & & & \\
\hline PE promedio & & 0.04 & & 0.04 & & & \\
\hline
\end{tabular}

Cuadro 6. Cuadrados medios, grados de libertad (gl) y significancia de la poliembrionía (PE) en maíz, evaluada en 16 combinaciones germoplásmicas en $\mathrm{F}_{2}$ y $\mathrm{RC}_{1}$, en condiciones de invernadero. Buenavista, Coah. 2009.

\begin{tabular}{lrcc}
\hline FV & $\mathrm{gl}$ & $\mathrm{F} 2$ & $\mathrm{RC}_{1}$ \\
\hline Repeticiones & 4 & 0.00002 & 0.0003 \\
Cruzas & 15 & $0.00085^{* *}$ & $0.0035^{* *}$ \\
Población 1 (D) & 7 & $0.00045^{* *}$ & 0.0017 \\
Población 2 (C) & 7 & $0.00127^{* *}$ & $0.0031^{* *}$ \\
Poblaciones (D vs. C) & 1 & 0.00072 & $0.0202^{* *}$ \\
Error experimental & 60 & 0.0002 & 0.0007 \\
CV (\%) & & 26.2 & 14.8 \\
PE promedio & & 0.05 & 0.018 \\
\hline
\end{tabular}

$\mathrm{CV}=$ coeficiente de variación; $* *$ significativo con $\mathrm{P} \leq 0.01$.

Cuadro 7. Valores de $\chi^{2}$ calculada y significancia por grupo segregante $\mathrm{F}_{2} \mathrm{o} \mathrm{RC}_{1}$, para invernadero y campo, en el análisis genético del carácter poliembrionía en maíz.

\begin{tabular}{|c|c|c|c|c|c|c|c|}
\hline \multirow[b]{2}{*}{ Grupos } & \multicolumn{2}{|c|}{$\begin{array}{c}\mathrm{F}_{2} \\
\text { Invernadero }\end{array}$} & \multicolumn{2}{|c|}{$\begin{array}{c}\mathrm{RC}_{1} \\
\text { Invernadero }\end{array}$} & \multicolumn{2}{|c|}{$\begin{array}{c}\mathrm{F}_{2} \\
\text { Campo }\end{array}$} & \multirow{2}{*}{$\begin{array}{c}\begin{array}{c}\mathrm{F}_{2} \\
\text { Campo }\end{array} \\
\mathrm{D}\end{array}$} \\
\hline & D & $\mathrm{C}$ & D & $\mathrm{C}$ & D & C & \\
\hline Com-1 & $15.7 * *$ & $15.2 * *$ & $49.2 * *$ & $12.7 * *$ & $72.5^{* *}$ & $35.0 * *$ & $36.4^{* *}$ \\
\hline Com-2 & 0.4 & 0.0 & $30.1 * *$ & $18.7 * *$ & $6.5^{*}$ & $39.8 * *$ & $7.3^{* *}$ \\
\hline Com 3 & $14.4 * *$ & $9.3^{* *}$ & $70.2 * *$ & $8.8^{* *}$ & nd & nd & nd \\
\hline Com-4 & 0.9 & 0.2 & $34.7 * *$ & $5.7 *$ & nd & nd & nd \\
\hline Com-5 & $10.5^{* *}$ & 1.2 & $56.8^{* *}$ & $47.9^{* *}$ & $20.6^{* *}$ & $21.7 * *$ & $30.5^{* *}$ \\
\hline Com-6 & $6.3^{*}$ & $8.3^{* *}$ & $16.4^{* *}$ & $39.4 * *$ & $34.8^{* *}$ & $17.0^{* *}$ & $34.1 * *$ \\
\hline Mat-1 & 2.5 & 0.1 & $37.3 * *$ & $13.7 * *$ & nd & nd & nd \\
\hline Mat-2 & $4.1^{*}$ & $8.4 * *$ & $42.3 * *$ & $5.4^{*}$ & nd & nd & nd \\
\hline
\end{tabular}

${ }^{\ddagger}$ Hipótesis $15: 1$ (en $\left.\mathrm{F}_{2}\right)$ y $12: 4\left(\right.$ en $\left.\mathrm{RC}_{1}\right) ;{ }^{*} \mathrm{y} * *=\mathrm{P} \leq 0.05$ y $\mathrm{P} \leq 0.01$ respectivamente. Para $\mathrm{F}_{2}, 1 \mathrm{gl}, \alpha 0.05=3.84$ y $\alpha 0.01=6.63$. Para RC1, 3 gl, $\alpha 0.05=7.81$ y $\alpha 0.01=11.34 ; \chi^{2}$ tabulada $=3.84$ y 6.64 , respectivamente; nd $=$ dato no disponible. 
Con base en los resultados de este trabajo, es pertinente señalar que la poliembrionía que aquí se alude es diferente a la de mutantes recesivos como la señalada por Kermicle $(1969 ; 1971)$ o Pilu (2000); de hecho, en una perspectiva histórica, la presente PE ha sido considerada de herencia cuantitativa (Espinoza et al., 1998). Sin embargo, la experiencia acumulada en la selección y su uso con fines prácticos, requiere de un replanteamiento sobre su control genético.

En este contexto, son tres las características que se tomaron en cuenta para este replanteamiento. Primera: en procesos habituales de autofecundaciones sucesivas, con la pretendida homocigosidad creciente (nivel $S_{3}$ ), no se ha logrado fijar el carácter PE. Segunda: los cruzamientos de $\quad \mathrm{C} \quad \mathrm{o} \quad \mathrm{D}$ con germoplasma exótico generan invariablemente una $F_{1}$ No-PE. Tercera: la recuperación de la PE se logra en progenies subsecuentes, pero en proporciones bajas. Así, con respecto a la propuesta de herencia de esta poliembrionía, la prueba de hipótesis aplicada por la vía de $\chi^{2}$ a los valores observados, sea en plántulas obtenidas en invernadero o en campo, de $\mathrm{F}_{2} \mathrm{o}$ $\mathrm{RC}_{1}$ (Cuadro 7), el modelo de herencia propuesto considera la acción de dos loci espistáticos en los cuales basta con presentar un alelo dominante en cualquiera de ellos para inducir normalidad en maíz y generar un embrión por semilla y una plántula en la germinación. De este modo, el fenómeno de poliembrionía sólo se presentará cuando los dos alelos de cada loci se encuentren en homocigosis recesiva.

En este trabajo, el análisis de $\mathrm{F}_{2} \mathrm{o} \mathrm{RC}_{1}$ hacia la fuente PE permitió detectar proporciones segregantes cercanas a dieciseisavos. Las pruebas estadísticas en grupos $\mathrm{F}_{2}$ en invernadero detectaron siete grupos germoplásmicos cuya segregación se ajustó a una proporción 15:1 (normal NoPE : mutante PE). Sin embargo, en la mayoría de casos en uno $\mathrm{u}$ otro nivel de germoplasma, hubo valores inferiores pero cercanos a las proporciones propuestas por el modelo de esta epistasis, sea de 15:1 en $\mathrm{F}_{2}$ o de 12:4 en $\mathrm{RC}_{1}$.

La proporción global de PE observada en los casos $\mathrm{F}_{2}$ indica que del valor esperado de $6.25 \%(15: 1)$ sólo se exhibió $4.0 \%$, mientras que en $\mathrm{RC}_{1}$ del valor esperado de $25 \%(12: 4)$ sólo se exhibió $17.5 \%$. Bajo las dos modalidades, esta PE encuentra obstáculos para su expresión, y es más notoria en los casos de dosis de germoplasma 50:50 (PE : No-PE) donde la penetrancia observada fue de $65 \%$, a diferencia del $70 \%$ registrado para los casos de 75:25; es decir, una mayor dosis de germoplasma poliembriónico favorece la expresión del carácter.

\section{CONCLUSIONES}

La poliembrionía en las dos poblaciones de maíz aquí evaluadas es controlada por dos loci epistáticos recesivos duplicados, donde basta la presencia de un alelo dominante de cualquiera de ellos para expresar la condición normal de planta. Así mismo, la poliembrionía encontrada en las poblaciones de maíz analizadas pudiera verse afectada por casos de penetrancia incompleta hasta de $50 \%$, en función del material externo no poliembriónico con el que se combine.

\section{AGRADECIMIENTOS}

Al Consejo Nacional de Ciencia y Tecnología (CONACYT) y al Programa de Fitomejoramiento e Instituto Mexicano del Maíz "Dr. Mario E. Castro Gil" de la Universidad Autónoma Agraria Antonio Narro, Saltillo, Coahuila, por la oportunidad otorgada al tesista para el desarrollo de sus estudios de maestría en ciencias en Fitomejoramiento.

\section{BIBLIOGRAFÍA}

CETENAL, Centro de Estudios del Territorio Nacional (1975) Carta Topográfica G14C33, escala 1:50 000. Saltillo, Coahuila. Secretaría de Programación y Presupuesto (SPP), Gobierno Federal de los Estados Unidos Mexicanos. México, D. F.

Erdelska O (1996) Polyembryony in maize-histological analysis. Acta Soc. Bot. Pol. 65:123-125.

Espinoza J, M C Vega, E Navarro, G A Burciaga (1998) Poliembrionía en maíces de porte normal enano. Agron. Mesoam. 9:83-88

Erdelska O, Z Vidovencova (1992) Cleavage polyembryony in maize. Sex. Plant Reprod. 5:224-226.

Evans M M S (2007) The indeterminate gametophyte 1 gene of maize encodes a LOB domain protein required for embryo sac and leaf development. The Plant Cell 19:46-62.

Hallauer A R, F O Miranda (1988) Quantitative Genetics in Maize Breeding. Iowa State University Press. USA. 468 p.

Kermicle J L (1969) Androgenesis conditioned by a mutation in maize. Science 166:1422-1424.

Kermicle J L (1971) Pleiotropic effects on seed development of the indeterminate gametophyte gene in maize. Am. J. Bot. 58:1-7.

Morgan D T Jr, R D Rappleye (1951) Polyembryony in maize and lily, following X-irradiation of the pollen. J. Hered. 42:91-93.

Pesev N, R Petrovic, Lj Zececiv, M Milosevic (1976) Study of possibility in raising maize inbred lines with two embryos. Theor. Appl. Genet. 47:197-201.

Pilu R (2000) The twin trait maize. Maize Gen. Coop. Newsl. 74:51.

SAS Institute (1999) SAS User's Guide. Statistics. Version 8.2. SAS Inst. Cary, NC. 102 p. 